\title{
Numerical Approximation of a Cauchy Problem for a Parabolic Partial Differential Equation
}

\author{
By Richard E. Ewing and Richard S. Falk*
}

\begin{abstract}
A procedure for the numerical approximation of the Cauchy problem for the following linear parabolic partial differential equation is defined:

$$
\begin{aligned}
& u_{t}-\left(p(x) u_{x}\right)_{x}+q(x) u=0, \quad 0<x<1,0<t \leqslant T ; \quad u(0, t)=f_{1}(t), \\
& 0<t \leqslant T ; \quad u(1, t)=f_{2}(t), \quad 0<t \leqslant T ; \quad p(0) u_{x}(0, t)=g(t), \\
& 0<t_{0} \leqslant t \leqslant T ; \quad|u(x, t)| \leqslant M, \quad 0 \leqslant x \leqslant 1,0 \leqslant t \leqslant T .
\end{aligned}
$$

The procedure involves Galerkin-type numerical methods for related parabolic initial boundary-value problems and a linear programming problem. Explicit a priori error estimates are presented for the entire discrete procedure when the data $f_{1}, f_{2}$, and $g$ are known only approximately.
\end{abstract}

1. Introduction. In many physical problems in heat conduction, the interior of the body is inaccessible for temperature measurements. Since it is then impossible to obtain an initial temperature distribution within the body, any approximations of the temperature distribution at later times must rely entirely upon data which can be measured at the boundary. For example, one can often use both measurements of temperature and heat flux at the boundary to compensate for the lack of an initial temperature distribution. An additional problem is that these boundary data are only accurate to within some prescribed measurement errors.

We shall obtain error estimates for the numerical approximation of a mathematical formulation of a class of problems of this type. Consider the numerical approximation of the solution of the following Cauchy problem for a linear parabolic partial differential equation:

Problem (P): Find a function $u=u(x, t)$ satisfying

(a) $\frac{\partial u}{\partial t}-\frac{\partial}{\partial x}\left(p(x) \frac{\partial u}{\partial x}\right)+q(x) u=0, \quad 0<x<1,0<t \leqslant T$,

(b) $u(0, t)=f_{1}(t), \quad 0<t \leqslant T$,

(c) $u(1, t)=f_{2}(t), \quad 0<t \leqslant T$,

(d) $p(0) \frac{\partial u}{\partial x}(0, t)=g(t), \quad 0<t_{0} \leqslant t \leqslant T$,

(e) $|u(x, t)| \leqslant M, \quad 0 \leqslant x \leqslant 1,0 \leqslant t \leqslant T$,

Received December 13, 1978; revised February 21, 1979.

AMS (MOS) subject classifications (1970). Primary 65M30, 65N30.

Key words and phrases. Cauchy problem, error estimates, improperly posed problem.

* This work was performed while the authors were at the University of Wisconsin-Madison Mathematics Research Center and was sponsored by the U. S. Army under contract \#DAAG2975-C-0024. The first author was also supported by U. S. Army contract \#DAAG29-78-G-0161 and NSF Grant \#MCS78-09525. The second author was also supported by NSF Grant \#MCS78-02737. 
where the data $f_{1}, f_{2}$, and $g$ are known only approximately as $f_{1}^{*}, f_{2}^{*}$, and $g^{*}$ such that
(a) $\left\|f_{1}-f_{1}^{*}\right\|_{[0, T]} \leqslant \epsilon_{0}$,
(b) $\left\|f_{2}-f_{2}^{*}\right\|_{[0, T]} \leqslant \epsilon_{0}$,
(c) $\left\|g-g^{*}\right\|_{\left[t_{0}, T\right]} \leqslant \epsilon_{0}$,

with $\epsilon_{0}>0$ and where for any function $f=f(t)$

$$
\|f\|_{[a, b]}=\sup _{a \leqslant t \leqslant b}|f(t)| .
$$

We assume that the following hypotheses are satisfied:

(H1) $f_{1}, f_{2}, g, p$, and $q$ are such that a classical solution $u$ to (1.1) exists.

(H2) The functions $p, p^{\prime}$ and $q$ are uniformly Hölder continuous in $0 \leqslant x \leqslant 1$ and satisfy

(a) $0<p_{*} \leqslant p(x) \leqslant p^{*}$,

(b) $0 \leqslant q_{*} \leqslant q(x) \leqslant q^{*}$,

(c) $\left|p^{\prime}(x)\right| \leqslant p^{\prime *}$.

(H3) $f_{1}$ and $f_{2}$ and their derivatives through order 2 are absolutely continuous on $[0, T]$ and $\partial^{3} f_{i} / \partial t^{3} \in L_{2}[0, T], i=1,2$. Denote by $K_{1}$ a constant satisfying

$$
\left\|f_{1}\right\|_{[0, T]}+\left\|f_{1}^{\prime}\right\|_{[0, T]}+\left\|f_{2}\right\|_{[0, T]}+\left\|f_{2}^{\prime}\right\|_{[0, T]}+\|g\|_{\left[t_{0}, T\right]} \leqslant K_{1} .
$$

(H4) For the Sturm-Liouville problem with eigenvalues and corresponding normalized eigenfunctions, satisfying

(a) $\left(p \varphi_{n}^{\prime}\right)^{\prime}-q \varphi_{n}+\lambda_{n} \varphi_{n}=0,0<x<1$,

(b) $\varphi_{n}(0)=\varphi_{n}(1)=0$,

there exists a $d>0$ such that

$$
d=\inf _{n}\left(\lambda_{n+1}-\lambda_{n}\right)
$$

(H5) The approximations (1.2) hold. In addition

(a) $\left\|f_{1}^{\prime}-f_{1}^{* \prime}\right\|_{[0, T]} \leqslant \epsilon_{0}$,

(b) $\left\|f_{2}^{\prime}-f_{2}^{* \prime \prime}\right\|_{[0, T]} \leqslant \epsilon_{0}$.

(H6) If $M>0$ is the constant given in (1.1(e)), we have

(a) $\left\|f_{1}\right\|_{[0, T]} \leqslant M$,

(b) $\left\|f_{2}\right\|_{[0, T]} \leqslant M$,

(c) $\left\|f_{1}^{*}\right\|_{[0, T]} \leqslant M$,

(d) $\left\|f_{2}^{*}\right\|_{[0, T]} \leqslant M$.

Without the extra assumption (1.1 (e)), the Cauchy problem (1.1 (a)-(d)) is not well-posed in the sense of Hadamard [3] - [8], [13] since the solution does not depend continuously upon the data. However, for the problem as stated with hypotheses (H1)-(H6) satisfied, a continuous dependence result was obtained in [6] .

Many studies of the continuous dependence of the Cauchy problem for various parabolic problems have appeared in the literature [3]-[8], [13]. Other formulations 
in terms of control problems have also appeared in the control theory literature [11], [12], [14]. In [7] Ginsberg considered numerical approximation of the Cauchy problem for the heat equation $u_{t}=u_{x x}$ with $g(t) \equiv 0$ in $(1.1(\mathrm{e}))$ by expanding the data in Fourier series and estimating Fourier coefficients. In [3], [4] Cannon and Douglas outlined numerical procedures for various Cauchy problems for the heat equation by reducing the problems to mathematical programming techniques. In [5] Cannon and one of the authors presented a direct numerical method for a slightly different Cauchy problem for the heat equation in which a Taylor series expansion for the data is numerically approximated. In [6] a numerical scheme requiring numerical approximation of several unknown eigenvalues and eigenfunctions was presented without explicit error estimates for the approximations. In this paper the numerical schemes involve only solution of linear parabolic initial boundary-value problems and a simple linear programming problem. More importantly, explicit a priori error estimates for the entire procedure are presented.

In Section 2, basic notation is presented and Problem (P) is reduced by linearity into two simple initial boundary-value problems and an optimization problem. In Section 3, Galerkin-type numerical schemes are defined for the initial boundary-value problems and a linear programming problem is formulated to solve the optimization problem. Several basic lemmas needed to prove the main result are stated in Section 4. Then a priori error estimates for obtaining approximations to (1.1) with approximate data satisfying (1.2) are stated and proved. Finally proofs of two of the technical lemmas are given.

2. Preliminaries. We shall first define some of the notations used for various norms throughout the paper. Recall that in Section 1 we used the notation that, for any function $f=f(t)$,

$$
\|f\|_{[a, b]} \equiv \sup _{a \leqslant t \leqslant b}|f(t)| .
$$

For functions $\psi=\psi(x)$ defined on $(0,1)$, we shall denote by $|\psi|_{\infty}$ the norm $\|\psi\|_{L^{\infty}(0,1)}$ and by $W^{m, \infty}$ ( $m$ a positive integer) the usual Sobolev space of functions with norm

$$
|\psi|_{m, \infty} \equiv \sum_{j=0}^{m}\left|\frac{\partial^{j} \psi}{\partial x^{j}}\right|_{\infty} .
$$

For real $s$, we further denote by $H^{s}$ the Sobolev space $W^{s, 2}$ of real-valued functions defined on $(0,1)$ and by $\|\psi\|_{s}$ its corresponding norm. We note that $L^{2}(0,1)$ will be denoted by $H^{0}$ and $\|\psi\|_{L^{2}(0,1)}$ by $\|\psi\|_{0}$. For definitions of the other spaces, we refer the reader to $[10]$.

Also, for $X$ a normed space with norm $\|\cdot\|_{X}$ and $u:[a, b] \rightarrow X$, we define

$$
\|u\|_{L^{2}(a, b ; X)}^{2} \equiv \int_{a}^{b}\|u(\cdot, t)\|_{X}^{2} d t \quad \text { and } \quad\|u\|_{L^{\infty}(a, b ; X)} \equiv \sup _{a \leqslant t \leqslant b}\|u(\cdot, t)\|_{X} .
$$

Finally, for convenience, we define a bilinear form 


$$
a(u, v) \equiv\left(p(\cdot) u_{x}, v_{x}\right)+(q(\cdot) u, v),
$$

where $(\cdot, \cdot)$ denotes the $L^{2}(0,1)$ inner product.

We shall next present a reformulation of Problem (P) on which our approximation scheme will be based. We first choose a function $\chi=\chi(x, t)$, depending linearly on $f_{1}, f_{2}$ and their derivatives, which satisfies

(a) $\quad \chi(0, t)=f_{1}(t), \quad \chi(1, t)=f_{2}(t), \quad 0 \leqslant t \leqslant T$,

(b) $|\chi(\cdot, 0)|_{\infty} \leqslant K^{*} \max _{\substack{i=1,2 \\ j=0, \ldots, J_{1}}}\left\{\left|\frac{\partial^{j} f_{i}}{\partial t^{j}}(0)\right|\right\}$,

(c) $|\chi(\cdot, t)|_{1, \infty} \leqslant K^{* *} \max _{\substack{i=1,2 \\ j=0, \ldots, J_{2}}}\left\{\left|f_{i}(t)\right|,\left|\frac{\partial^{j} f_{i}}{\partial t^{j}}(0)\right|\right\}, \quad 0 \leqslant t \leqslant T$, and

(d) $\left|\frac{\partial \chi}{\partial t}(\cdot t)-\frac{\partial}{\partial x}\left(p \frac{\partial \chi}{\partial x}\right)(\cdot, t)+q \chi(\cdot, t)\right|_{\infty}$

$$
\leqslant K^{* * *} \max _{\substack{i=1,2 \\ j=0, \ldots, J_{2} \\ l=0, \ldots, J_{3}}}\left\{\frac{\partial^{l} f_{i}}{\partial t^{l}}(t),\left|\frac{\partial^{j} f_{i}}{\partial t^{j}}(0)\right|\right\}, \quad 0 \leqslant t \leqslant T,
$$

where $K^{*}, K^{* *}$ and $K^{* * *}$ are positive constants, $0 \leqslant J_{1} \leqslant 1,0 \leqslant J_{2} \leqslant 2$, and $0 \leqslant J_{3} \leqslant 1$. If $\chi$ is chosen so that it is necessary that $J_{2}=2$ for $(2.0(\mathrm{c}))$ or $(2.0(\mathrm{~d}))$ to be satisfied, we shall require the additional hypothesis

$$
\left|\frac{\partial^{2} f_{i}}{\partial t^{2}}(0)-\frac{\partial^{2} f_{i}^{*}}{\partial t^{2}}(0)\right| \leqslant \epsilon_{0}, \quad i=1,2 .
$$

One choice of $\chi$ (denoted $\tilde{\chi})$ which satisfies (2.0) with $J_{1}=0, J_{2}=0, J_{3}=1$, $K^{*}=1, K^{* *}=2$, and $K^{* * *}=1+2 p^{*}+q^{*}$ is given by

$$
\tilde{\chi}(x, t)=(1-x) f_{1}(t)+x f_{2}(t), \quad 0 \leqslant x \leqslant 1,0 \leqslant t \leqslant T .
$$

We shall also consider different choices of $\chi$ later which make full use of conditions $(2.0(\mathrm{c}))$ and $(2.0(\mathrm{~d}))$.

We next define a function $w=w(x, t)$ satisfying the initial boundary-value problem
(a) $\frac{\partial w}{\partial t}-\frac{\partial}{\partial x}\left(p \frac{\partial w}{\partial x}\right)+q w=F(x, t), \quad 0<x<1,0<t \leqslant T$,
(b) $w(0, t)=0, \quad 0 \leqslant t \leqslant T$,
(c) $w(1, t)=0, \quad 0 \leqslant t \leqslant T$,
(d) $w(x, 0)=0,0<x<1$, 
where

$$
F(x, t)=-\frac{\partial \chi}{\partial t}+\frac{\partial}{\partial x}\left(p \frac{\partial \chi}{\partial x}\right)-q \chi
$$

with $\chi$ satisfying (2.0). Also, for each $\psi \in L^{2}(0,1)$, we define a function $z^{\psi}$ satisfying the initial boundary-value problem:

$$
\begin{aligned}
& \text { (a) } \frac{\partial z^{\psi}}{\partial t}-\frac{\partial}{\partial x}\left(p \frac{\partial z^{\psi}}{\partial x}\right)+q z^{\psi}=0, \quad 0<x<1,0<t \leqslant T, \\
& \text { (b) } z^{\psi}(0, t)=0, \quad 0<t \leqslant T, \\
& \text { (c) } z^{\psi}(1, t)=0, \quad 0<t \leqslant T, \\
& \text { (d) } z^{\psi}(x, 0)=\psi, \quad 0<x<1 .
\end{aligned}
$$

Using $M$ from (1.1(d)), let

$$
K \equiv\left\{\varphi \in C^{0}[0,1]:|\varphi|_{\infty} \leqslant M \text { and } \varphi(0)=f_{1}(0), \varphi(1)=f_{2}(0)\right\}
$$

We can now reformulate Problem (P) using (2.1)-(2.3) and linearity of the operator in (1.1) as follows:

Find $\varphi$ (corresponding to $u(x, 0)$ from (1.1)) with $\varphi \in K$ such that

$$
p(0) \frac{\partial z^{\varphi-r}}{\partial x}(0, t)=g(t)-p(0) \frac{\partial w}{\partial x}(0, t)-p(0) \frac{\partial \chi}{\partial x}(0, t) \equiv G(t)
$$

where

$$
r(x) \equiv \chi(x, 0) .
$$

To see that this reformulation is equivalent to Problem (P), observe that, using linearity, the function $u^{\psi}=w+z^{\psi}+\chi$ satisfies
(a) $\frac{\partial u^{\psi}}{\partial t}-\frac{\partial}{\partial x}\left(p \frac{\partial u^{\psi}}{\partial x}\right)+q u^{\psi}=0, \quad 0<x<1,0<t \leqslant T$,
(b) $u^{\psi}(0, t)=f_{1}(t), \quad 0<t \leqslant T$,
(c) $u^{\psi}(1, t)=f_{2}(t), \quad 0<t \leqslant T$,
(d) $u^{\psi}(x, 0)=\psi+r, \quad 0<x<1$,
(e) $p(0) u_{x}^{\psi}(0, t)=p(0) w_{x}(0, t)+p(0) z_{x}^{\psi}(0, t)+p(0) \frac{\partial \chi}{\partial x}(0, t), \quad t_{0} \leqslant t \leqslant T$.

Choosing $\psi=\varphi-r$, we see that $u^{\varphi-r}$ solves Problem (P) provided we can find a $\varphi$ satisfying (2.4). (We note that since $\left|f_{1}(t)\right| \leqslant M$ and $\left|f_{2}(t)\right| \leqslant M, 0 \leqslant t \leqslant T$, the maximum principle implies that the condition $|\varphi|_{\infty} \leqslant M$ is equivalent to requiring that $|u(x, t)| \leqslant M$, for $0 \leqslant x \leqslant 1,0 \leqslant t \leqslant T$.)

Using this notation, we can now state a form of the continuous dependence result (proved in [6]) which we shall need later in the derivation of the error estimates. 
Lemma 1. Under hypotheses (H1)-(H6), there exist computable constants $C_{1}$ and $\gamma(0<\gamma<1)$ such that for all $\psi$ with $\mid \psi \mathrm{i}_{\infty} \leqslant M$,

$$
\left\|z^{\psi}\right\|_{L^{\infty}\left(t_{0}, T ; W^{1, \infty}\right)} \leqslant C_{1}\left\|z_{x}^{\psi}(0, \cdot)\right\|_{\left.t_{0}, T\right]}^{\gamma},
$$

where $z^{\psi}$ is the solution of (2.3).

3. Description of the Numerical Approximations. In this section we consider the problem of numerically approximating the solution of (1.1) (or equivalently, its reformulation described in Section 2) subject to the restriction (1.2). The restriction (1.2) comes from the fact that data measurement error is, in general, accurate only to within some measurement tolerance $\epsilon_{0}$. We denote by $\chi^{*}$ the function chosen in exactly the same way as $\chi$ with $f_{i}$ replaced by $f_{i}^{*}, i=1,2$, in (2.0). Let $F^{*}$ denote the function defined as $F$ in (2.2) with $\chi$ replaced by $\chi^{*}$. Then define $w^{*}$ as the solution of (2.1) with $F$ replaced by $F^{*}$,

$$
r^{*} \equiv \chi^{*}(x, 0)
$$

and

$$
G^{*}(t) \equiv g^{*}(t)-p(0) \frac{\partial w^{*}}{\partial x}(0, t)-p(0) \frac{\partial \chi^{*}}{\partial x}(0, t)
$$

We note here that from $(\mathrm{H} 3),(\mathrm{H} 5),(1.2),(2.0),(2.5)$, and (3.1), we have

$$
\begin{aligned}
& \text { (a) }|r|_{\infty} \leqslant K^{*} K_{1}, \\
& \text { (b) }\left|r^{*}\right|_{\infty} \leqslant K^{*}\left(K_{1}+\epsilon_{0}\right), \\
& \text { (c) }\left\|r-r^{*}\right\|_{0} \leqslant\left|r-r^{*}\right|_{\infty} \leqslant K^{*} \epsilon_{0} .
\end{aligned}
$$

Since the data is only known approximately as described above, we now define an approximation scheme based on a finite-dimensional analogue of Eq. (2.4) with $G$ replaced by $G^{*}$ from (3.2). We first describe schemes for obtaining approximations to $w$ and $z^{\psi}$ (assuming $\psi$ is known).

Let $S_{h}^{\beta}$ denote the space of continuous piecewise polynomials of degree $\beta-1$ defined on a uniform mesh of width $h$ on $[0,1]$ and vanishing at $x=0$ and $x=1$. We shall consider a family of such spaces for $0<h<1$. We assume each space in this family satisfies the following so-called "inverse assumptions": $u \in S_{h}^{\beta}$ implies that for some constant $C_{2}$,

$$
\begin{aligned}
& \text { (a) }|u|_{1, \infty} \leqslant C_{2} h^{-1}|u|_{\infty}, \\
& \text { (b) }|u|_{\infty} \leqslant C_{2} h^{-1 / 2}\|u\|_{0} .
\end{aligned}
$$

We also assume that our test spaces $S_{h}^{\beta}$ satisfy the following approximation assumptions: If $u \in S_{h}^{\beta}$ and $I_{h} u$ is the interpolate of $u$ in $S_{h}^{\beta}$, then for some constant $C_{3}$,

$$
\begin{aligned}
& \text { (a) }\left\|u-I_{h} u\right\|_{0} \leqslant C_{3} h^{s}\|u\|_{s}, \quad s \geqslant 0, \\
& \text { (b) }\left|I_{h} u\right|_{1, \infty} \leqslant C_{3}|u|_{1, \infty} .
\end{aligned}
$$


Let $k>0$ be the stepsize in time, $N_{T}=T / k \in Z, t^{n}=n k$, and $\varphi_{n}=\varphi\left(t^{n}\right)$. We shall present a Crank-Nicolson-Galerkin approximation for $w$, the solution of (2.1)(2.2). Define $W:\left\{0=t_{0}, t_{1}, \ldots, t_{N_{T}}=T\right\} \rightarrow S_{h_{1}}^{4}$ by

$$
\left(\frac{W_{n+1}-W_{n}}{k}, V\right)+a\left(\frac{W_{n+1}+W_{n}}{2}, V\right)=(F(\cdot,(n+1 / 2) k), V)
$$

for all $V \in S_{h_{1}}^{4}$ and $n=0,1, \ldots, N_{T}-1$, with $W_{0} \equiv 0$, where $a(\cdot, \cdot)$ is defined in Section 2. We similarly define $W_{n}^{*}$ to be the analogous approximation to $w^{*}(n k)$ given by (2.1)-(2.2) with $F$ replaced by $F^{*}$.

The scheme defined in (3.6) is known to have a time-truncation error of the order $k^{2}$. We shall use another $O\left(k^{2}\right)$ time-stepping method with better stability properties but greater work estimates to approximate $z$, the solution of (2.3) with $\psi$ assumed known. Define $Z^{\psi}:\left\{0=t_{0}, t_{1}, \ldots, t_{N_{T}}=T\right\} \rightarrow S_{h}^{2}$ by

$$
\begin{aligned}
& \left(\frac{Z_{n+\alpha}^{\psi}-Z_{n}^{\psi}}{\alpha k}, v\right)+a\left(Z_{n+\alpha}^{\psi}, v\right)=0, \\
& \left(\frac{Z_{n+1}^{\psi}-Z_{n}^{\psi}}{\alpha k}, v\right)+a\left(Z_{n+1}^{\psi}, v\right)=\frac{-(1-\alpha)}{\alpha} a\left(Z_{n+\alpha}^{\psi}, v\right),
\end{aligned}
$$

with

$$
\left(Z_{0}^{\psi}, v\right)=(\psi, v)
$$

for all $v \in S_{k}^{2}$ where $\alpha=1-\sqrt{2} / 2$.

We note that since each of the time-stepping schemes defined above have $O\left(k^{2}\right)$ time-discretization error but different spatial orders of approximation, we shall use the time step $k$ to tie the two approximations together. Thus, $k$ will be the same in each of (3.6) and (3.7). We shall then see from Lemmas 3 and 4 below that in order to balance the temporal and spatial discretization errors in each problem separately, we shall let $h=k$ in the definition of $Z$ and $h_{1}=k^{1 / 2}$ in the definition of $W$.

Let $N_{\mathbf{0}}=\| t_{0} / k \rrbracket+1$ where $\| \tilde{r} \rrbracket$, for $\tilde{r} \in \mathbf{R}$, is the greatest integer less than $\tilde{r}$. Using the above definitions, we can now define an approximate problem as follows:

Problem $\left(\mathrm{P}_{A}\right)$ : Find $\varphi_{h} \in K_{h}$ such that

$$
J\left(\varphi_{h}\right)=\inf _{\psi_{h} \in K_{h}} J\left(\psi_{h}\right)
$$

where

(a) $K_{h}=\left\{\varphi_{h} \in S_{h}^{2}:\left|\varphi_{h}\right|_{\infty} \leqslant M, \varphi_{h}(0)=f_{1}^{*}(0)\right.$, and $\left.\varphi_{h}(1)=f_{2}^{*}(0)\right\}$,

(b) $J\left(\psi_{n}\right)=\max _{n=N_{0}, \ldots, N_{T}} \mid g^{*}(n k)-p(0) \frac{\partial \chi^{*}}{\partial x}(0, n k)$

$$
-p(0) \frac{\partial}{\partial x} W_{n}^{*}(0)-p(0) \frac{\partial}{\partial x} Z_{n}^{\psi_{h}{ }^{-r^{*}}}(0) \mid
$$


We then take as our approximation to $u(t)$ at $t=n k$ the function

$$
U_{n}=W_{n}^{*}+Z_{n}^{\varphi_{h}-r^{*}}+\chi^{*}(\cdot, n k)
$$

We now show how Problem $\left(\mathrm{P}_{A}\right)$ can be solved by linear programming. Let $h$ be such that $H=1 / h \in Z$ and

$$
\psi_{h}=\sum_{i=1}^{H-1} C_{i} \Phi_{i}+f_{1}^{*}(0) \Phi_{0}+f_{2}^{*}(0) \Phi_{H},
$$

where

$$
\Phi_{i}=\left\{\begin{array}{l}
0, \quad x \leqslant(i-1) h, \\
x / h-(i-1), \quad(i-1) h \leqslant x \leqslant i h, \\
1+i-x / h, \quad i h \leqslant x \leqslant(i+1) h, \\
0, \quad x \geqslant(i+1) h .
\end{array}\right.
$$

Note that the constraint $\left|\psi_{h}\right|_{\infty} \leqslant M$ is equivalent to $\left|C_{i}\right| \leqslant M, i=1, \ldots, H-1$, and $\left|f_{i}^{*}(0)\right| \leqslant M, i=1,2$. Then, by linearity,

$$
\frac{\partial}{\partial x} Z_{n}^{\psi_{h}{ }^{-r^{*}}}(0)=\sum_{i=1}^{H-1} C_{i} \frac{\partial}{\partial x} Z_{n}^{\Phi_{i}}(0)+\frac{\partial}{\partial x} Z_{n}^{f_{1}^{*}(0) \Phi_{0}+f_{2}^{*}(0) \Phi_{H}}(0)-\frac{\partial}{\partial x} Z_{n}^{r^{*}}(0)
$$

Hence Problem $\left(\mathrm{P}_{A}\right)$ can be written as:

Find $\vec{C}=\left(C_{1}, \ldots, C_{H-1}\right)$ minimizing $\lambda$ subject to the constraints

(a) $-M \leqslant C_{i} \leqslant M, i=1, \ldots, H-1$, and

(b) $-\lambda \leqslant g^{*}(n k)-p(0) \frac{\partial \chi^{*}}{\partial x}(0, n k)-p(0) \frac{\partial}{\partial x} W_{n}^{*}(0)$

$$
\begin{aligned}
& -p(0)\left[\sum_{i=1}^{H-1} C_{i} \frac{\partial}{\partial x} Z_{n}^{\Phi_{i}}(0)+\frac{\partial}{\partial x} Z_{n}^{\left.f_{1}^{*}(0) \Phi_{0}+f_{2}^{*}(0) \Phi_{H}(0)-\frac{\partial}{\partial x} Z_{n}^{r^{*}}(0)\right]}\right. \\
& \leqslant \lambda, \quad n=N_{0}, \ldots, N_{T} .
\end{aligned}
$$

4. Main Results. In order to derive our main result, we shall need several lemmas about the regularity and approximation of the solutions of (2.1)-(2.2) and (2.3) and the stability of problem (3.6). We shall now state these lemmas. The first lemma relates the smoothness of the solution of (2.3) to its initial smoothness and can be found in [2].

Lemma 2. Let $Z^{\psi}$ be the solution of problem (2.3). Then for $0<t_{0} \leqslant t$ and $s \geqslant 0$, there exists a constant $C_{4}$ such that

$$
\left\|Z^{\psi}(\cdot t)\right\|_{s} \leqslant C_{4} t_{0}^{-s / 2}\|\psi\|_{0} .
$$

LEMMA 3 (CF. WHEELER [15]). Let $w$ be the solution of (2.1)-(2.2) and $\left\{W_{n}\right\}$ be its approximation given by (3.6). If $w \in L^{\infty}\left(0, T ; W^{4, \infty}\right), \partial w / \partial t \in L^{2}\left(0, T ; H^{4}\right)$, and $\partial^{3} w / \partial t^{3} \in L^{2}\left(0, T ; H^{0}\right)$, then there are constants $C_{4}$ and $k_{0}>0$ such that for all 
$0<k<k_{0}$, we have for $n=0, \ldots, N_{T}$,

$$
\begin{array}{r}
\left|w(\cdot, n k)-W_{n}\right|_{\infty} \leqslant C_{4}\left\{h_{1}^{4}\left[\|w\|_{L^{\infty}\left(0, T ; w^{4, \infty}\right)}+\left\|\frac{\partial w}{\partial t}\right\|_{L^{2}\left(0 . T ; H^{4}\right)}\right]\right. \\
\left.+k^{2}\left\|\frac{\partial^{3} w}{\partial t^{3}}\right\|_{L^{2}\left(0, T ; H^{0}\right)}\right\} .
\end{array}
$$

We remark that sufficient conditions for $w$ to have the regularity required by Lemma 3 are that
(a) $F_{t t} \in L^{2}\left(0, T ; H^{0}\right)$,
(b) $F(x, 0)=0$ for $x=0$ and $x=1$, and
(c) $\frac{\partial}{\partial x}\left(p \frac{\partial F(x, 0)}{\partial x}\right)-q F(x, 0)-F_{t}(x, 0)=0 \quad$ for $x=0,1$,

where $F$ is given in (2.2). For the choice of $\chi$ denoted by $\tilde{\chi}$ above, (H3) implies (4.3(a)). However $(4.3(\mathrm{~b}))$ and $(4.3(\mathrm{c}))$ require special relationships to hold between various combinations of

$$
\frac{\partial^{j} f_{i}}{\partial t^{j}}(0), \frac{\partial^{l} p}{\partial x^{l}}(0), \frac{\partial^{l} p}{\partial x^{l}}(1), \frac{\partial^{j} q}{\partial x^{j}}(0), \text { and } \frac{\partial^{j} q}{\partial x^{j}}(1)
$$

for $i=1,2, l=0,1,2,3$ and $j=0,1,2$. If these conditions are not met by the given $p, q$, and $f_{i}, i=1,2$, then we must make another choice of $\chi$ for the results to apply. Another possible choice of $\chi$ which satisfies (2.0) and fits into our analysis is given by $\bar{\chi}$, which for each $t$ in $0 \leqslant t \leqslant T$ satisfies the boundary-value problem

$$
\begin{aligned}
& \text { (a) }-\frac{\partial}{\partial x}\left(p \frac{\partial}{\partial x} \bar{\chi}\right)=c(x, t) \equiv a_{1}+a_{2} t+2\left[b_{1} x+b_{2} t x\right], \\
& \text { (b) } \bar{\chi}(0, t)=f_{1}(t), \quad \bar{\chi}(1, t)=f_{2}(t),
\end{aligned}
$$

for specific constants $a_{1}, a_{2}, b_{1}$, and $b_{2}$.

We note that $\bar{\chi}$ is defined by

$$
\bar{\chi}(x, t)=f_{1}(t)-\alpha(t) \int_{0}^{x} \frac{d s}{p(s)}-\left(a_{1}+a_{2} t\right) \int_{0}^{x} \frac{s d s}{p(s)}-\left(b_{1}+b_{2} t\right) \int_{0}^{x} \frac{s^{2} d s}{p(s)}
$$

with

$$
\alpha(t)=\left[f_{1}(t)-f_{2}(t)-\left(a_{1}+a_{2} t\right) \int_{0}^{1} \frac{s d s}{p(s)}-\left(b_{1}+b_{2} t\right) \int_{0}^{1} \frac{s^{2} d s}{p(s)}\right] / \int_{0}^{1} \frac{d s}{p(s)}
$$

and satisfies (2.0) with $J_{1}=1, J_{2}=2, J_{3}=1$ and easily computable $K^{*}, K^{* *}$ and $K^{* * *} . \bar{\chi}$ is much more complicated than the choice $\tilde{\chi}$, but if we make the choices 


$$
\begin{aligned}
a_{1} & =-q(0) f_{1}(0)-f_{1}^{\prime}(0), \\
b_{1}= & {\left[-q(1) f_{2}(0)-f_{2}^{\prime}(0)-a_{1}\right] / 2, } \\
\alpha(0)= & {\left[f_{1}(0)-f_{2}(0)-a_{1} \int_{0}^{1} \frac{s d s}{p(s)}-b_{1} \int_{0}^{1} \frac{s^{2} d s}{p(s)}\right] / \int_{0}^{1} \frac{d s}{p(s)}, } \\
a_{2}=1 / 2\left\{\left[p(0) q^{\prime \prime}(0)+p^{\prime}(0) q^{\prime}(0)\right] f_{1}(0)-q(0) f_{1}^{\prime}(0)-f_{1}^{\prime \prime}(0)-a_{1} q(0)\right. & \left.-2 q^{\prime}(0) \alpha(0)+2 b_{1} p^{\prime}(0)\right\}, \\
b_{2}=1 / 4\left\{\left[p(1) q^{\prime \prime}(1)+p^{\prime}(1) q^{\prime}(1)\right] f_{2}(0)-q(1) f_{2}^{\prime}(0)-f_{2}^{\prime \prime}(0)-2 a_{2}\right. & \left.\quad-\left(a_{1}+2 b_{1}\right) q(1)+2 b_{1} p^{\prime}(1)+2 q^{\prime}(1)\left[\alpha(0)+a_{1}+b_{1}\right]\right\},
\end{aligned}
$$

then the compatibility assumptions (4.3) are satisfied without any special relationships holding between the functions $p, q$, and $f_{i}, i=1,2$. We remark that if $\partial^{2} q(0) / \partial x^{2}$ and $\partial^{2} q(1) / \partial x^{2}$ do not exist as required in the definition of $\bar{\chi}$, another $\chi$ should be chosen which would eliminate the extra smoothness assumption on $q$. In this case, choose $\hat{\chi}$ to satisfy the boundary-value problem

$$
\begin{aligned}
& -\frac{\partial}{\partial x}\left(p \frac{\partial}{\partial x} \hat{\chi}\right)+q \hat{\chi}=\hat{a}_{1}+\hat{a}_{2} t+\hat{b}_{1} x+\hat{b}_{2} t x, \\
& \hat{\chi}(0, t)=f_{1}(t), \quad \hat{\chi}(1, t)=f_{2}(t),
\end{aligned}
$$

with $\hat{a}_{1}, \hat{a}_{2}, \hat{b}_{1}$, and $\hat{b}_{2}$ chosen so that (4.3) is satisfied. We note that with this choice of $\chi$, the solution of a different boundary-value problem must be computed for each discrete time step to determine $F$.

When (4.3) is satisfied, we have by standard a priori estimates that for $n=$ $0, \ldots, N_{T}$,

$$
\left|w(\cdot, n k)-w_{n}\right|_{\infty} \leqslant C_{5}\left\{h_{1}^{4}+k^{2}\right\}
$$

where $C_{5}$ depends only upon the data $f_{1}, f_{2}, p$, and $q$. Using the inverse properties (3.4) satisfied by the subspaces $S_{h_{1}}^{4}$, we easily obtain the following result by a standard technique.

Corollary (3.1). There exists a constant $C_{6}$ such that for $n=0, \ldots, N_{T}$,

$$
\left|w(\cdot, n k)-w_{n}\right|_{1, \infty} \leqslant \frac{C_{6}}{h_{1}}\left[h_{1}^{4}+k^{2}\right] .
$$

Our next lemma allows us to obtain $L^{\infty}$-estimates for the error in approximation of (2.3) by (3.7) for times bounded away from $t=0$ (i.e., $0<t_{0} \leqslant t$ ).

LEMMA 4 (CF. BAKER ET AL. [1]). Let $z^{\psi}$ be the solution of (2.3) and $\left\{Z_{n}^{\psi}\right\}$ the approximation given by (3.7). Then there exist constants $C_{7}=C_{7}\left(t_{0}\right)$ such that for $n=N_{0}, \ldots, N_{T}$,

$$
\left|z^{\psi}(\cdot, n k)-Z_{n}^{\psi}\right|_{\infty} \leqslant C_{7}\left\{h^{2}+k^{2}\right\}\|\psi\|_{0} .
$$


Again, using our inverse assumptions on $S_{h}^{2}$, we obtain

Corollary (4.1). There exists a constant $C_{8}$ such that for $n=N_{0}, \ldots, N_{T}$,

$$
\left|z^{\psi}(\cdot, n k)-Z_{n}^{\psi}\right|_{1, \infty} \leqslant \frac{C_{8}}{h}\left[h^{2}+k^{2}\right]\|\psi\|_{0}
$$

We shall also require a special stability result for the approximation scheme (3.6). Since the proof of the following lemma is quite technical, we shall defer it until after the proof of our main result.

Lemma 5. Let $\left\{W_{n}\right\}$ be the solution of (3.6). If for some constant $\tau_{0}>0$, we restrict $k$ and $h_{1}$ such that $k h_{1}^{2} \leqslant \tau_{0}$, then there is a constant $C_{9}$ such that

$$
\left|W_{n}\right|_{1, \infty} \leqslant C_{9}\|F\|_{L^{\infty}\left(0, T ; H^{0}\right)} .
$$

The last lemma which we shall state gives an a priori estimate for the linear programming problem defined in (3.15). Again the proof of this lemma will be deferred until after the proof of our main result.

LEMma 6. Let $\varphi_{h} \in K_{h}$ be the solution of the linear programming problem (3.15). Then there exists a constant $C_{10}$ such that

$$
\begin{array}{r}
\max _{n=N_{0}, \cdots, N_{T}}\left|g^{*}(n k)-p(0) \frac{\partial \chi^{*}}{\partial x}(0, n k)-p(0) \frac{\partial}{\partial x} W_{n}^{*}(0)-p(0) \frac{\partial}{\partial x} Z_{n}^{\varphi_{h}-r^{*}}(0)\right| \\
=J\left(\varphi_{h}\right) \leqslant C_{10}\left\{\epsilon_{0}+h+\left[h_{1}^{4}+k^{2}\right] / h_{1}+\left[h^{2}+k^{2}\right] / h\right\} .
\end{array}
$$

We are now in a position to state our major result and prove it using Lemmas 1-6.

THEOREM 1. Let $u$ be the solution of Problem $(\mathrm{P})$ and $\left\{U_{n}\right\}$ be the approximation defined by Problem $\left(\mathrm{P}_{A}\right)$ and (3.11). Suppose that hypotheses $(\mathrm{H} 1)-(\mathrm{H} 6)$ are satisfied, that $F$ (defined by (2.2)) satisfies the regularity conditions (4.3) and that $\varphi=$ $u(x, 0)$ satisfies $\|\varphi\|_{1} \leqslant C_{11}$ for some constant $C_{11}>0$. If the mesh sizes $k, h$, and $h_{1}$ are chosen to satisfy $k=h=h_{1}^{2}$, then there exists a constant $C_{12}$ which is independent of $k$ such that for $n=N_{0}, \ldots, N_{T}$,

$$
\left|u(\cdot, n k)-U_{n}\right|_{1, \infty} \leqslant C_{12}\left[\epsilon_{0}+k\right]^{\gamma},
$$

where $\epsilon_{0}$ and $\gamma$ are the constants defined by (H5) and (2.7), respectively.

Proof. In the reformulation of Problem (P), we wrote the solution as

$$
u=u^{\varphi-r}=w+z^{\varphi-r}+\chi .
$$

From (3.11) we have

$$
U_{n}=W_{n}^{*}+Z_{n}^{\varphi_{h}-r^{*}}+\chi^{*}(\cdot, n k) .
$$

Using the triangle inequality, we obtain

$$
\begin{aligned}
\left|u(; n k)-U_{n}\right|_{1, \infty} \leqslant & \left|w(; n k)-W_{n}\right|_{1, \infty}+\left|W_{n}-W_{n}^{*}\right|_{1, \infty} \\
& +\left|z^{\varphi-r}(\cdot n k)-Z_{n}^{\varphi_{h}-r^{*}}\right|_{1, \infty}+\left|\chi(; n k)-\chi^{*}(; n k)\right|_{1, \infty} .
\end{aligned}
$$


The first term on the right of (4.13) is bounded using (4.5) as follows:

$$
\left|w(\cdot, n k)-W_{n}\right|_{1, \infty} \leqslant C_{6}\left[h_{1}^{4}+k^{2}\right] / h_{1} .
$$

From (1.2), (H5), (H7), and (2.0), we see that

$$
\left|\chi(\cdot n k)-\chi^{*}(; n k)\right|_{1, \infty} \leqslant 2 K^{*} \epsilon_{0} .
$$

Since $k=h_{1}^{2}$ by hypothesis, we can use Lemma 5, (1.2), (2.0(d)), (2.2), (H5) and possibly (H7) to bound the second term on the right side of (4.13). We obtain

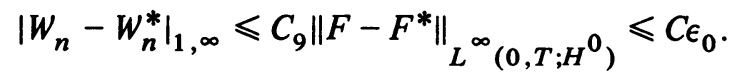

In order to treat the third term on the right of (4.13) we use the triangle inequality,

$$
\begin{aligned}
& \left|z^{\varphi-r}(\cdot, n k)-Z_{n}^{\varphi_{h}-r^{*}}\right|_{1, \infty} \\
& \quad \leqslant\left|z^{\varphi-r}(; n k)-z^{\varphi_{h}-r^{*}}(; n k)\right|_{1, \infty}+\left|z^{\varphi_{h}-r^{*}}(; n k)-Z_{n}^{\varphi_{h}-r^{*}}\right|_{1, \infty} .
\end{aligned}
$$

Using (3.3(b)), (3.10(a)), and (4.7) we have for $n=N_{0}, \ldots, N_{T}$,

$$
\left|z^{\varphi_{h}-r^{*}}(\cdot n k)-Z_{n}^{\varphi_{h}-r^{*}}\right|_{1, \infty} \leqslant \frac{C_{8}}{h}\left[h^{2}+k^{2}\right]\left\|\varphi_{h}-r^{*}\right\|_{0}
$$

$$
\leqslant \frac{C_{8}}{h}\left[h^{2}+k^{2}\right]\left\{\left|\varphi_{h}\right|_{\infty}+\left|r^{*}\right|_{\infty}\right\} \leqslant C\left[h^{2}+k^{2}\right] / h
$$

Combining the above estimates, we obtain

$$
\begin{aligned}
\left|u(\cdot n k)-U_{n}\right|_{1, \infty} \leqslant & C\left\{\epsilon_{0}+\left[h_{1}^{4}+k^{2}\right] / h_{1}+\left[h^{2}+k^{2}\right] / h\right\} \\
& +\left|z^{\varphi-r}(; n k)-z^{\varphi_{h}-r^{*}}(; n k)\right|_{1, \infty} .
\end{aligned}
$$

In order to estimate the last term on the right of (4.19), we shall use the continuous dependence result from (2.7). We obtain

$$
\left|z^{\varphi-r}(\cdot n k)-z^{\varphi_{h}-r^{*}}(\cdot n k)\right|_{1, \infty} \leqslant C_{1}\left\|z_{x}^{\varphi-r}(0, \cdot)-z_{x}^{\varphi_{h}-r^{*}}(0, \cdot)\right\|_{\left\{t_{0}, T\right]}^{\gamma}
$$

For any function $y(t) \in W^{2, \infty}\left[t_{0}, T\right]$, we now define $I_{k} y$ to be the piecewise linear interpolate of $y$ on the time mesh of width $k$. Then we obtain (using properties of a linear interpolate)

$$
\begin{gathered}
\left\|z_{x}^{\varphi-r}(0, \cdot)-z_{x}^{\varphi_{h}-r^{*}}(0, \cdot)\right\|_{\left[t_{0}, T\right]} \\
(4.21) \leqslant \\
\quad\left\|z_{x}^{\varphi-r}(0, \cdot)-I_{k} z_{x}^{\varphi-r}(0, \cdot)\right\|_{\left[t_{0}, T\right]}+\left\|I_{k} z_{x}^{\varphi_{h}-r^{*}}(0, \cdot)-z_{x}^{\varphi_{h}-r^{*}}(0, \cdot)\right\|_{\left[t_{0}, T\right]} \\
\quad+\max _{n=N_{0}, \ldots, N}\left|z_{x}^{\varphi-r}(0, n k)-z_{x}^{\varphi_{h}-r^{*}}(0, n k)\right| .
\end{gathered}
$$

By standard results in approximation theory, the first two terms on the right of (4.21) 
are bounded by

$$
C k^{2}\left[\left\|z_{x t t}^{\varphi-r}(0, \cdot)\right\|_{\left[t_{0}, T\right]}+\left\|z_{x t t}^{\varphi_{h}-r^{*}}(0, \cdot)\right\|_{\left[t_{0}, T\right]}\right] .
$$

Next, differentiating Eq. (2.3(a)) and using Lemma 2 and the Sobolev lemma, we obtain for any $\psi \in H^{0}$,

$$
\left\|z_{x t t}^{\psi}(0, \cdot)\right\|_{[0, T]} \leqslant C\left\|z^{\psi}(\cdot, t)\right\|_{6} \leqslant C\|\psi\|_{0} \text { for } 0<t_{0} \leqslant t \leqslant T .
$$

Combining the above estimates and using (3.3) and (3.10(a)) to see that $\|\varphi-r\|_{0}$ and $\left\|\varphi_{h}-r^{*}\right\|_{0}$ are bounded, we obtain

$$
\begin{aligned}
\left\|z_{x}^{\varphi-r}(0, \cdot)-z_{x}^{\varphi_{h}-r^{*}}(0, \cdot)\right\|_{\left[t_{0}, T\right]} \\
\leqslant C k^{2}+\max _{n=N_{0}, \ldots, N_{T}}\left|z_{x}^{\varphi-r}(0, n k)-z_{x}^{\varphi_{h}-r^{*}}(0, n k)\right| \\
\leqslant C k^{2}+\max _{n=N_{0}, \ldots, N_{T}}\left|\frac{\partial}{\partial x} z^{\varphi-r}(0, n k)-\frac{\partial}{\partial x} Z_{n}^{\varphi_{h}-r^{*}}(0)\right| \\
+\max _{n=N_{0}, \ldots, N_{T}}\left|\frac{\partial}{\partial x} Z_{n}^{\varphi_{h}-r^{*}}(0)-\frac{\partial}{\partial x} z^{\varphi_{h}-r^{*}}(0, n k)\right|
\end{aligned}
$$

Using (3.3), (3.10), and (4.7), we estimate the last term on the right of (4.24) by

$$
\begin{aligned}
\max _{n=N_{0}, \ldots, N_{T}} \mid \frac{\partial}{\partial x} Z_{n}^{\varphi_{h}-r^{*}}(0) & -\frac{\partial}{\partial x} z^{\varphi_{h}-r^{*}}(0, n k) \mid \\
& \leqslant \frac{C_{8}}{h}\left[h^{2}+k^{2}\right]\left\|\varphi_{h}-r^{*}\right\|_{0} \leqslant C\left[h^{2}+k^{2}\right] / h
\end{aligned}
$$

Next, using (2.4) and the triangle inequality, we see that the second term on the right of (4.24) is bounded by

$$
\begin{aligned}
& \max _{n=N_{0}, \ldots, N_{T}}\left|\frac{\partial}{\partial x} z^{\varphi-r}(0, n k)-\frac{\partial}{\partial x} Z_{n}^{\varphi_{h}-r^{*}}(0)\right| \\
& \leqslant \max _{n=N_{0}, \ldots, N_{T}}\left|\frac{g(n k)}{p(0)}-\frac{\partial w}{\partial x}(0, n k)-\frac{\partial \chi}{\partial x}(0, n k)-\frac{\partial}{\partial x} Z_{n}^{\varphi_{h}-r^{*}}(0)\right| \\
& \leqslant \max _{n=N_{0}, \ldots, N_{T}}\left\{\frac{1}{p(0)}\left|g(n k)-g^{*}(n k)\right|+\left|\frac{\partial w}{\partial x}(0, n k)-\frac{\partial}{\partial x} W_{n}(0)\right|\right. \\
& \left.+\left|\frac{\partial}{\partial x} W_{n}(0)-\frac{\partial}{\partial x} W_{n}^{*}(0)\right|+\left|\frac{\partial \chi}{\partial x}(0, n k)-\frac{\partial \chi^{*}}{\partial x}(0, n k)\right|\right\} \\
& +\max _{n=N_{0}, \ldots, N_{T}} \frac{1}{p(0)} \mid g^{*}(n k)-p(0) \frac{\partial}{\partial x} W_{n}^{*}(0)-p(0) \frac{\partial \chi^{*}}{\partial x}(0, n k) \\
& \equiv \text { term }_{1}+\operatorname{term}_{2} .
\end{aligned}
$$


Using (4.5), (4.15) and (4.16), we can bound the first term on the right of (4.26). We obtain

$$
\operatorname{term}_{1} \leqslant C\left\{\epsilon_{0}+\left[h_{1}^{4}+k^{2}\right] / h_{1}\right\} .
$$

We note from (3.9) and (3.10) that the second term on the right of (4.26) is just $p(0)^{-1}$ times $J\left(\varphi_{h}\right)$ from our Problem $\left(\mathrm{P}_{A}\right)$. We then use Lemma 6 to obtain the a priori estimate

$$
\operatorname{term}_{2}=J\left(\varphi_{h}\right) / p(0)
$$

$$
\leqslant C_{10}\left\{\epsilon_{0}+h+\left[h_{1}^{4}+k^{2}\right] / h_{1}+\left[h^{2}+k^{2}\right] / h\right\} .
$$

Then combining (4.19), (4.20), and (4.24)-(4.28), we see that for $n=N_{0}, \ldots, N_{T}$,

$$
\left|u(\cdot, n k)-U_{n}\right|_{1, \infty} \leqslant C\left\{\epsilon_{0}+h+\left[h_{1}^{4}+k^{2}\right] / h_{1}+\left[h^{2}+k^{2}\right] / h\right\}^{\gamma} .
$$

Then choosing $h=k$ and $h_{1}=k^{1 / 2}$, we obtain

$$
\left|u(\cdot, n k)-U_{n}\right|_{1, \infty} \leqslant C\left\{\epsilon_{0}+k\right\}^{\gamma}
$$

for $n=N_{0}, \ldots, N_{T}$, which was to be proved.

Next, assume that the linear programming problem described in Section 3 is solved to within the tolerance $J\left(\varphi_{h}\right) \leqslant \sigma_{1}$ for some $\sigma_{1}>0$. Replacing the estimate (4.28) by the above inequality, we obtain the following error estimate.

Corollary T1. Assume the hypotheses of Theorem 1 are satisfied. If $\varphi_{h}^{*}=$ $\Sigma_{i=1}^{H-1} C_{i}^{*} \Phi_{i}$, the solution of the linear programming problem defined by (3.15), satisfies $J\left(\varphi_{h}^{*}\right) \leqslant \sigma_{1}$, then for $n=N_{0}, \ldots, N_{T}$ we have for some constant $C_{13}>0$,

$$
\left|u(\cdot, n k)-U_{n}\right|_{1, \infty} \leqslant C_{13}\left[\epsilon_{0}+k+\sigma_{1}\right]^{\gamma} .
$$

We shall finally give proofs for Lemmas 5 and 6 which were stated previously.

Proof of Lemma 5. To prove this lemma we will need to make use of results from elliptic regularity theory, spectral theory in Hilbert spaces, and the theory of interpolation spaces. We shall assume the reader is familiar with these concepts, since to provide detailed explanations would unduly lengthen the proof. In order to simplify the exposition, we first introduce some additional notation. Let $Q$ be the solution operator for the two-point boundary-value problem

$$
\begin{aligned}
& \text { (a) }-\frac{\partial}{\partial x}\left(p \frac{\partial y}{\partial x}\right)+q y=f, \quad 0<x<1 \\
& \text { (b) } y(0)=0 \\
& \text { (c) } y(1)=0
\end{aligned}
$$

(i.e., $y=Q f$ ). Let $Q_{h_{1}}$ be the solution operator for the Galerkin approximation of (4.31). Then $y_{h_{1}}=Q_{h_{1}} f$ is defined by

$$
a\left(y_{h_{1}}, v_{h_{1}}\right)=\left(f, v_{h_{1}}\right), \quad v_{h_{1}} \in S_{h_{1}}^{4} .
$$

Now, set $L_{h_{1}}=Q_{h_{1}}^{-1}$ (i.e., the inverse of $Q_{h_{1}}$ on $S_{h_{1}}^{4}$ ). For normed spaces $X$ and $Y$, 
let $L(X, Y)$ denote the space of linear operators from $X$ to $Y$ and $\|\cdot\|_{L(X, Y)}$ the (operator) norm in this space. We shall first establish that

$$
\left|W_{n}\right|_{1, \infty} \leqslant C\left\|L_{h_{1}}^{7 / 8} W_{n}\right\|_{0}
$$

We note that

$$
\begin{aligned}
\left|W_{n}\right|_{1, \infty}= & \left|Q_{h_{1}} L_{n_{1}} W_{n}\right|_{1, \infty} \leqslant\left|\left(Q_{h_{1}}-Q\right) L_{n_{1}} W_{n}\right|_{1, \infty}+\left|Q L_{n_{1}} W_{n}\right|_{1, \infty} \\
\leqslant & \left|Q_{n_{1}} L_{n_{1}} W_{n}-I_{n_{1}} Q L_{n_{1}} W_{n}\right|_{1, \infty}+\left|I_{n_{1}} Q L_{n_{1}} W_{n}-Q L_{n_{1}} W_{n}\right|_{1, \infty} \\
& +\left|Q L_{n_{1}} W_{n}\right|_{1, \infty}
\end{aligned}
$$

where $I_{h_{1}}$ is the interpolation operator mapping $H_{0}^{1} \rightarrow S_{h_{1}}^{4}$. By the inverse properties of $S_{h_{1}}^{4}$ given in (3.4), we see that

$$
\begin{aligned}
& \left|Q_{h_{1}} L_{h_{1}} W_{n}-I_{h_{1}} Q L_{h_{1}} W_{n}\right|_{1, \infty} \\
& \quad \leqslant C h_{1}^{-3 / 2}\left\|Q_{h_{1}} L_{h_{1}} W_{n}-I_{h_{1}} Q L_{h_{1}} W_{n}\right\|_{0} \\
& \quad \leqslant C h_{1}^{-3 / 2}\left\{\left\|Q_{h_{1}} L_{h_{1}} W_{n}-Q L_{h_{1}} W_{n}\right\|_{0}+\left\|Q L_{n_{1}} W_{n}-I_{n_{1}} Q L_{n_{1}} W_{n}\right\|_{0}\right\} .
\end{aligned}
$$

Using standard properties of the Galerkin approximation, we note that the first term on the right of (4.35) can be estimated as follows:

$$
h_{1}^{-3 / 2}\left\|\left(Q_{h_{1}}-Q\right) L_{h_{1}} W_{n}\right\|_{0} \leqslant C h_{1}^{-3 / 2} h_{1}^{7 / 4}\left\|Q L_{h_{1}} W_{n}\right\|_{7 / 4}
$$

Also, using (3.5 (a)), we obtain

$$
h_{1}^{-3 / 2}\left\|Q L_{h_{1}} W_{n}-I_{h_{1}} Q L_{h_{1}} W_{n}\right\|_{0} \leqslant C h_{1}^{-3 / 2} h_{1}^{7 / 4}\left\|Q L_{h_{1}} W_{n}\right\|_{7 / 4}
$$

Then, using (3.5(b)) and the Sobolev lemma, we see that

$$
\begin{gathered}
\left|I_{h_{1}} Q L_{n_{1}} W_{n}-Q L_{n_{1}} W_{n}\right|_{1, \infty} \leqslant\left|I_{h_{1}} Q L_{n_{1}} W_{n}\right|_{1, \infty}+\left|Q L_{n_{1}} W_{n}\right|_{1, \infty} \\
\leqslant\left(C_{3}+1\right)\left|Q L_{n_{1}} W_{n}\right|_{1, \infty} \leqslant C\left\|Q L_{n_{1}} W_{n}\right\|_{7 / 4} .
\end{gathered}
$$

We then collect terms in the above inequalities and use a standard a priori estimate for elliptic problems (i.e., $\left.Q \in L\left(H^{-1 / 4}, H^{7 / 4}\right)[10]\right)$ to obtain

$$
\left|W_{n}\right|_{1, \infty} \leqslant C\left\|Q L_{n_{1}} W_{n}\right\|_{7 / 4} \leqslant C\left\|L_{n_{1}} W_{n}\right\|_{-1 / 4},
$$

where the last norm is defined below in (4.41). Then, in order to obtain (4.33) we need only show that

$$
\left\|L_{h_{1}} W_{n}\right\|_{-1 / 4} \leqslant C\left\|L_{h_{1}}^{7 / 8} W_{n}\right\|_{0} .
$$

Let $P: L^{2} \rightarrow S_{h_{1}}^{4}$ be the $L^{2}$ projection operator into $S_{h_{1}}^{4}$. Note that $L_{h_{1}}$ is selfadjoint, so $L_{h_{1}}^{1 / 8}$ is also selfadjoint. Then we see that 


$$
\begin{aligned}
\left\|L_{n_{1}} W_{n}\right\|_{-1 / 4} & \equiv \sup _{v \in C_{0}^{\infty}} \frac{\left(L_{h_{1}} W_{n}, v\right)}{\|v\|_{1 / 4}}=\sup _{v \in C_{0}^{\infty}} \frac{\left(L_{h_{1}} W_{n}, P v\right)}{\|v\|_{1 / 4}} \\
& =\sup _{v \in C_{0}^{\infty}} \frac{\left(L_{h_{1}^{7}}^{7 / 8} W_{n}, L_{n_{1}}^{1 / 8} P v\right)}{\|v\|_{1 / 4}} \leqslant\left\|L_{h_{1}^{7 / 8}} W_{n}\right\|_{0} \sup _{v \in C_{0}^{\infty}} \frac{\left\|L_{h_{1}}^{1 / 8} P v\right\|_{0}}{\|v\|_{1 / 4}}
\end{aligned}
$$

Next, since we have that

$$
\left\|L_{h_{1}}^{1 / 2} P v\right\|_{0}=[a(P v, P v)]^{1 / 2} \leqslant C\|P v\|_{1}
$$

and

$$
\left\|L_{h_{1}}^{0} P u\right\|_{0} \leqslant\|P v\|_{0}
$$

we can use an interpolation theorem due to Heinz [9] (compare also with [10]) to establish that

$$
\left\|L_{h_{1}^{1 / 8}}^{1 / 8} P\right\|_{0} \leqslant C\|P U\|_{1 / 4}
$$

Then, since approximation properties of $S_{h_{1}}^{4}$ yield

$$
\|P u\|_{1 / 4} \leqslant C\|v\|_{1 / 4},
$$

we can combine (4.41), (4.44) and (4.45) to obtain

$$
\left\|L_{h_{1}} W_{n}\right\|_{-1 / 4} \leqslant C\left\|L_{h_{1}^{7 / 8}}^{7} W_{n}\right\|_{0}
$$

Now, to establish (4.8) we solve a set of first-order difference equations arising from (3.6) to obtain

$$
\begin{aligned}
W_{n}=k \sum_{j=0}^{n-1}\left[\left\{\left[I+\frac{k}{2} L_{n_{1}}\right]^{-1}\left[I-\frac{k}{2} L_{n_{1}}\right]\right\}^{j}\right. \\
\left.\cdot\left[I+\frac{k}{2} L_{n_{1}}\right]^{-1} \operatorname{PF}\left(\cdot\left[n-\frac{1}{2}-j\right] k\right)\right] .
\end{aligned}
$$

Then we see that

$$
\left\|L_{h_{1}}^{7 / 8} W_{n}\right\|_{0} \leqslant\left\|k L_{h_{1}}^{7 / 8}\left[I+\frac{k}{2} L_{h_{1}}\right]^{-1} P F\left(\cdot\left[n-\frac{1}{2}\right] k\right)\right\|_{0}
$$

$$
\begin{aligned}
& +k \sum_{j=1}^{n-1} \| L_{h_{1}}^{7 / 8}\left\{\left[I+\frac{k}{2} L_{h_{1}}\right]^{-1}\left[I-\frac{k}{2} L_{h_{1}}\right]\right\} \\
& \cdot\left[I+\frac{k}{2} L_{h_{1}}\right]^{-1} \|_{L\left(H^{0}, H^{0}\right)} \\
& \cdot\left\|P F\left(\cdot\left[n-\frac{1}{2}-j\right] k\right)\right\|_{0}
\end{aligned}
$$


For the first term on the right of (4.48), we obtain

$$
\begin{aligned}
& \left\|k L_{h_{1}}^{7 / 8}\left[I+\frac{k}{2} L_{h_{1}}\right]^{-1} P F\left(\cdot\left[n-\frac{1}{2}\right] k\right)\right\|_{0} \\
& \leqslant 2^{7 / 8} k^{1 / 8}\left\|\left(\frac{k}{2}\right)^{7 / 8} L_{h_{1}^{7 / 8}}^{7}\left[I+\frac{k}{2} L_{h_{1}}\right]^{-1}\right\|_{L\left(H^{0}, H^{0}\right)}\left\|P F\left(\cdot\left[n-\frac{1}{2}\right] k\right)\right\|_{0} \\
& \leqslant 2^{7 / 8} k^{1 / 8} \sup _{0<\lambda<\infty}\left\{\frac{\lambda^{7 / 8}}{1+\lambda}\right\}\left\|P F\left(\cdot\left[n-\frac{1}{2}\right] k\right)\right\|_{0} \\
& \left.\leqslant 2\left\|P F\left(\cdot\left[n-\frac{1}{2}\right] k\right)\right\|_{0} \quad \text { (for } k \leqslant 2\right) .
\end{aligned}
$$

Since it is known that the eigenvalues $\Lambda_{i}$ of $L_{h_{1}}$ satisfy $0<\Lambda_{i} \leqslant \beta_{0} h_{1}^{-2}$, the mesh ratio restriction $k h_{1}^{-2} \leqslant \tau_{0}$ contained in the statement of Lemma 5 implies that

$$
\Lambda_{i} \leqslant \beta_{0} \tau_{0} / k
$$

From [1], we know that there exist constants $C_{0}$ and $K_{0}$ such that for $j=1,2, \ldots$, and $0<\lambda \leqslant \beta_{0} \tau_{0} / k$,

$$
\left|\frac{1-k \lambda / 2}{1+k \lambda / 2}\right|^{j} \leqslant K_{0} e^{-C_{0}(k / 2) \lambda j} .
$$

Then, since clearly $e^{-C x} x^{7 / 8}$ is bounded for all $x \geqslant 0$, we use (4.51) to see that

$$
\begin{gathered}
\left\|L_{h_{1}}^{7 / 8}\left\{\left[I+\frac{k}{2} L_{h_{1}}\right]^{-1}\left[I-\frac{k}{2} L_{h_{1}}\right]\right\}^{j}\left[I+\frac{k}{2} L_{h_{1}}\right]^{-1}\right\|_{L\left(H^{0}, H^{0}\right)} \\
\leqslant \sup _{0<\lambda<\beta_{0} \tau_{0} / k}\left|\frac{1-k \lambda / 2}{1+k \lambda / 2}\right|^{j} \frac{\lambda^{7 / 8}}{1+k \lambda / 2} \leqslant \sup _{0<\lambda \leqslant \beta_{0} \tau_{0} / k} e^{-C_{0}(k / 2) \lambda j} \lambda^{7 / 8} \\
\leqslant \sup _{0<\lambda<\beta_{0} \tau_{0} / k} e^{-C_{0}(k / 2) \lambda j} \frac{(k \lambda j / 2)^{7 / 8}}{(j k / 2)^{7 / 8}} \leqslant C_{12}(j k / 2)^{-7 / 8} .
\end{gathered}
$$

Thus, combining the above estimates, we have

$$
\begin{aligned}
\left\|L_{h_{1}}^{7 / 8} W_{n}\right\|_{0} & \leqslant 2\left\|P F\left(\cdot\left[n-\frac{1}{2}\right] k\right)\right\|_{0}+\|P F\|_{L^{\infty}\left(0, T ; H^{0}\right)} k \sum_{j=1}^{n-1} \frac{C_{12}}{(j k / 2)^{7 / 8}} \\
& \leqslant\|P F\|_{L^{\infty}\left(0, T ; H^{0}\right)}\left[2+\frac{C_{12}}{2^{7 / 8}} \int_{0}^{T} \frac{1}{t^{7 / 8}} d t\right] \\
& \leqslant\|P F\|_{L^{\infty}\left(0, T ; H^{0}\right)}\left[2+\frac{8 C_{12}}{2^{7 / 8}} T^{1 / 8}\right] \leqslant C\|F\|_{L^{\infty}\left(0, T ; H^{0}\right)}
\end{aligned}
$$

Finally, combining (4.39), (4.46) and (4.53) proves Lemma 5. 
Proof of Lemma 6. From (H5), (3.9), (3.10), (4.5), (4.15), and (4.16) we see that for all $\psi_{h} \in K_{h}$

$$
\begin{aligned}
& J\left(\varphi_{h}\right) \leqslant J\left(\psi_{h}\right) \\
& =\max _{n=N_{0}, \ldots, N_{T}}\left|g^{*}(n k)-p(0) \frac{\partial \chi^{*}}{\partial x}(0, n k)-p(0) \frac{\partial}{\partial x} W_{n}^{*}(0)-p(0) \frac{\partial}{\partial x} Z_{n}^{\psi}{ }^{-r^{*}}(0)\right| \\
& \leqslant \max _{n=N_{0}, \ldots, N_{T}}\left\{\left|g^{*}(n k)-g(n k)\right|+p(0)\left|\frac{\partial \chi^{*}}{\partial x}(0, n k)-\frac{\partial \chi}{\partial x}(0, n k)\right|\right. \\
& +p(0)\left|\frac{\partial}{\partial x} W_{n}^{*}(0)-\frac{\partial}{\partial x} W_{n}(0)\right|+p(0)\left|\frac{\partial}{\partial x} W_{n}(0)-\frac{\partial w}{\partial x}(0, n k)\right| \\
& \left.+p(0)\left|\frac{\partial}{\partial x} Z_{n}^{\psi} n^{-r^{*}}(0)-\frac{\partial}{\partial x} z^{\varphi-r}(0, n k)\right|\right\} \\
& \leqslant C\left[\epsilon_{0}+\left(h_{1}^{4}+k^{2}\right) / h_{1}\right]+\max _{n=N_{0}, \ldots, N_{T}} p(0)\left|\frac{\partial}{\partial x} Z_{n}^{\psi} h^{-r^{*}}(0)-\frac{\partial}{\partial x} z^{\varphi-r}(0, n k)\right| .
\end{aligned}
$$

Now

$$
\begin{aligned}
& \left|\frac{\partial}{\partial x} Z_{n}^{\psi h^{-r^{*}}}(0)-\frac{\partial}{\partial x} z^{\varphi-r}(0, n k)\right|
\end{aligned}
$$

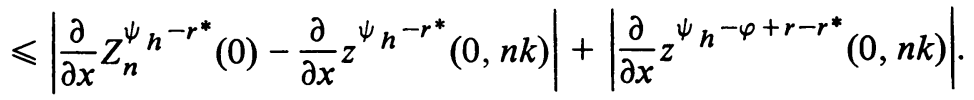

From (4.7), (3.3), and (3.10) we see that for $n=N_{0}, \ldots, N_{T}$,

$$
\begin{aligned}
\left|\frac{\partial}{\partial x} Z_{n}^{\psi h^{-r^{*}}}(0)-\frac{\partial}{\partial x} z^{\psi_{h^{-r^{*}}}}(0, n k)\right| & \leqslant \frac{C_{8}}{h}\left[h^{2}+k^{2}\right]\left\|\psi_{h}-r^{*}\right\|_{0} \\
& \leqslant C\left[h^{2}+k^{2}\right] / h .
\end{aligned}
$$

Next using the Sobolev lemma, Lemma 2, and (3.3(c)), we obtain for $n=N_{0}, \ldots, N_{T}$, and for any $\psi_{k} \in K_{h}$,

$$
\begin{aligned}
\left|\frac{\partial}{\partial x} z^{\psi_{h}-\varphi+r-r^{*}}(0, n k)\right| & \leqslant\left\|z^{\psi_{h}-\varphi+r-r^{*}}\right\|_{2} \\
& \leqslant C\left\|\psi_{h}-\varphi+r-r^{*}\right\|_{0} \leqslant C\left[\epsilon_{0}+\left\|\psi_{h}-\varphi\right\|_{0}\right] .
\end{aligned}
$$

Choose $\psi_{h}$ such that

(a) $\psi_{h}($ ih $)=\varphi($ ih $), \quad i=1, \ldots, H-1$,

(b) $\psi_{h}(0)=f_{1}^{*}(0)$,

(c) $\psi_{h}(1)=f_{2}^{*}(0)$.

Then $\psi_{h} \in K_{h}$ and $\psi_{h}$ is almost equal to $I_{h} \varphi$, the piecewise linear interpolate of $\varphi$. 
Then from (3.5),

$$
\left\|\psi_{h}-\varphi\right\|_{0} \leqslant\left\|\psi_{h}-I_{h} \varphi\right\|_{0}+\left\|I_{h} \varphi-\varphi\right\|_{0} \leqslant\left\|\psi_{h}-I_{h} \varphi\right\|_{0}+C h\|\varphi\|_{1} .
$$

But we see that

$$
\psi_{h}-I_{h} \varphi= \begin{cases}0, & h \leqslant x \leqslant(H-1) h \\ \left(1-\frac{x}{h}\right)\left[f_{1}^{*}(0)-f_{1}(0)\right], & 0 \leqslant x \leqslant h \\ {\left[\frac{x}{h}-(H-1)\right]\left[f_{2}^{*}(0)-f_{2}(0)\right],} & (H-1) h \leqslant x \leqslant 1\end{cases}
$$

so we have

$$
\left\|\psi_{h}-I_{h} \varphi\right\|_{0} \leqslant C \epsilon_{0} .
$$

Combining (4.54)-(4.61), we obtain

$$
J\left(\varphi_{h}\right) \leqslant C\left[\epsilon_{0}+h+\left(h_{1}^{4}+k^{2}\right) / h_{1}+\left(h^{2}+k^{2}\right) / h\right] .
$$

Acknowledgement. The authors wish to thank Peter Sammon for several helpful discussions regarding the proof of Lemma 5.

Department of Mathematics

The Ohio State University

Columbus, Ohio 43210

Department of Mathematics

Rutgers University

New Brunswick, New Jersey 08903

1. G. A. BAKER, J. H. BRAMBLE \& V. THOMÉE, "Single step Galerkin approximations for parabolic problems," Math. Comp., v. 31, 1977, pp. 818-847.

2. J. H. BRAMBLE, A. H. SCHATZ, V. THOMÉE \& L. B. WAHLBIN, "Some convergence estimates for semidiscrete Galerkin type approximations for parabolic equations," SIAM J. Numer. Anal., v. 14, 1977, pp. 218-241.

3. J. R. CANNON, "A Cauchy problem for the heat equation," Ann. Mat. Pura Appl., v. 66, 1964, pp. $155-166$.

4. J. R. CANNON \& JIM DOUGLAS, JR., "The Cauchy problem for the heat equation," SIAM J. Numer. Anal., v. 4, 1967, pp. 317-336.

5. J. R. CANNON \& R. E. EWING, "A direct numerical procedure for the Cauchy problem for the heat equation," J. Math. Anal. Appl., v. 56, 1976, pp. 7-17.

6. R. E. EWING, "The Cauchy problem for a linear parabolic partial differential equation," J. Math. Anal. Appl. (To appear.)

7. F. GINSBERG, "On the Cauchy problem for the one-dimensional heat equation," Math. Comp., v. 17, 1963, pp. 257-269.

8. J. HADAMARD, Lectures on the Cauchy Problem in Linear Partial Differential Equations, Yale Univ. Press, New Haven, Conn., 1923.

9. E. HEINZ, "Beiträge zur Störungstheorie der Spektralzerlegung," Math. Ann., v. 123, 1951, pp. $415-438$.

10. J. L. LIONS \& E. MAGENES, Non-homogeneous Boundary Value Problems and Applications, Vol. I, Springer-Verlag, New York, 1972.

11. R. C. MACCAMY, V. J. MIZEL \& T. I. SEIDMAN, "Approximate boundary controllability for the heat equation," J. Math. Anal. Appl., v. 23, 1968, pp. 699-703 and II, J. Math. Anal. Appl., v. 28, 1969, pp. 482-492. 
12. V. J. MIZEL \& T. I. SEIDMAN, "Observation and prediction for the heat equation," J. Math. Anal. Appl., v. 28, 1969, pp. 303-312, and II, J. Math. Anal. Appl., v. 38, 1972, pp. 149-166.

13. C. PUCCI, "Alcuni limitazioni per le soluzioni di equazioni parabiliche," Ann. Mat. Pura Appl., v. 48, 1959, pp. 161-172.

14. T. I. SEIDMAN, "Observation and prediction for one-dimensional diffusion equations," J. Math. Anal. Appl., v. 51, 1975, pp. 165-175.

15. M. F. WHEELER, " $L_{\infty}$ estimates of optimal order for Galerkin methods for one dimensional second order parabolic and hyperbolic equations," SIAM J. Numer. Anal., v. 10, 1973, pp. 908-913. 